Original Research

\title{
Mechano-Vibrational Spectroscopy of Tissues and Materials Using Vibrational Optical Coherence Tomography: A New Non-Invasive and Non-Destructive Technique
}

\author{
Frederick H. Silver ${ }^{*}$, Nikita Kelkar, Tanmay Deshmukh, Istvan Horvath, Ruchit G. Shah
}

OptoVibronex, LLC., Allentown, Pa, USA; E-Mails: fhsilver@hotmail.com; nuk1@scaletmail.rutgers.com; tmdeshmu@usc.edu; dr.horvath.istvan@gmail.com; ruchit.shah27@gmail.com

* Correspondence: Frederick H. Silver; E-Mail: fhsilver@hotmail.com

Academic Editor: Hossein Hosseinkhani

Special Issue: $\underline{\text { Applications and Development of Biomaterials in Medicine }}$

Recent Progress in Materials

2020 , volume 2 , issue 2

doi:10.21926/rpm.2002010
Received: February 04, 2020

Accepted: April 20, 2020

Published: April 29, 2020

\begin{abstract}
Elastic moduli of tissues and synthetic polymeric materials are important design properties needed to develop new implants. In this paper we report the use of vibrational optical coherence tomography (VOCT) to measure the resonant frequency and calculate the modulus of tissues and synthetic polymers non-invasively and non-destructively in vitro and in vivo.

Values of tissue and polymer moduli were obtained by applying an audible sinusoidal sound wave to the surface of each specimen. The sound wave travels to the interior of the material and is reflected back to the surface from underlying layers. A spectrum of resonant frequencies and moduli are then obtained. By analyzing the frequencies at which the maximum displacements are observed the resonant frequencies are determined of the material components and the moduli can then be calculated from calibration equations.

The characteristic spectrum of resonant frequencies measured is a finger print by which the composition of the underlying tissue components can be determined using VOCT. The resonant frequencies of cells and tissues range from about $40 \mathrm{~Hz}$ for fat to about $990 \mathrm{~Hz}$ for
\end{abstract}

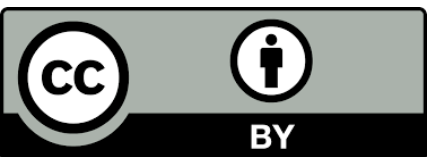

(C) 2020 by the author. This is an open access article distributed under the conditions of the Creative Commons by Attribution License, which permits unrestricted use, distribution, and reproduction in any medium or format, provided the original work is correctly cited. 
lamellar bone. The modulus for these tissues ranges from about $1 \mathrm{MPa}$ (cells) to that for extracellular matrix components that range from 2 to $173 \mathrm{MPa}$ (tissue macromolecular components).

Measurements reported on synthetic polymers indicate that the resonant frequency ranges from $80 \mathrm{~Hz}$ (silicone rubber) to $2800 \mathrm{~Hz}$ (acrylonitrile butadiene styrene) and values of the modulus are found to be between $2 \mathrm{MPa}$ and $2120 \mathrm{MPa}$, respectively. The resonant frequency and modulus are shown to decrease as a result of fatigue in Viton rubber. It is concluded that the width of the resonant frequency peak is related to the molecular weight distribution and that VOCT may serve as method to rapidly determine molecular weight distributions and properties after cyclic loading of uncrosslinked and crosslinked polymers.

\section{Keywords}

Tissues; polymers; implant materials; mechanical properties; elastic modulus; vibrational optical coherence tomography; resonant frequency; molecular weight distributions

\section{Introduction}

Natural materials have gained widespread use as replacements for damaged tissues in a wide variety of applications. Allografts and xenografts have grown appreciably in their use in applications that include: bladder regeneration, burns, breast reconstruction, facial cosmetic defects, free flap coverage, hernias, ophthalmic defects, oral defects, replacement of cardiovascular tissues, tendon/ligament repair, and vaginal repair [1-16]. Synthetic polymeric devices have also been used as replacements in the repair of rotator cuff, Achilles and quadriceps tendons, primary staged and revised breast reconstruction, hernia repair, treatment of chronic wounds such as diabetic foot ulcers and pulmonary patches [16, 17]. Synthetic polymers including poly(dimethyl siloxane), poly(ethylene), poly(tetrafluoroethylene), poly(methyl methacrylate), poly(urethanes) and poly(olefins) are the primary synthetic materials used to replace the function of both soft and hard tissues [17]. The properties of natural and synthetic materials do not exactly match those of the tissues and organs that they are used to replace. However, it is important to avoid stress concentration and cellular hyperplasia at the tissue-implant interface [18]. Modulus mismatches result in cellular hyperplasia and eventual failure of the implant-tissue interface due to upregulation of mechanotransduction [18]. For these reasons, it is important to be able to measure the modulus of both implants and tissues non-invasively and non-destructively in vivo [19].

In addition to understanding the effects of modulus mismatches at the host tissue-implant interface, the mechanical properties of cells and tissues are reported to reflect changes associated with diseases such as connective tissue disorders, Osteognesis Imperfecta, Marfan's Syndrome, Ehlers Danlos Syndrome, Scleroderma and cancer [20-25]. These diseases are characterized by changes in the mechanical properties such as modulus and hardness. Therefore, it is important to be able to accurately calculate the value of the modulus of tissues and implants in vivo, not only to better design implants and artificial organs, but also to provide early diagnoses of connective tissue disorders and cancer [20-27]. 
In principle, measurement of tissue modulus would appear to be a straight forward problem to solve. However, this measurement is complicated by the fact that tissues behave in a viscoelastic manner and have mechanical properties that are non-linear, strain-rate and time dependent, and depend on the value of the applied stress and strain. This makes mechanical characterization of tissues complex even outside of the body (in vitro) and very difficult when they within the body (in vivo).

A number of tests have been used to characterize tissues and implants including constant rateof-strain experiments where the modulus $(E)$ is obtained from the slope of the stress-strain curve. This test does not require assuming a value of Poisson's ratio. It requires mounting the sample ends in grips and is usually conducted until the sample fails in tension. The determination of the stiffness or modulus requires measurements made at several different levels of the strain; the slope (E) depends on the rate of deformation. As the sample is stretched at higher strain-rates, the modulus appears to increase because the sample does not have time to relax during deformation. This test can be repeated many times during fatigue testing in tension and compression. The limitations to this test are: (a) the sample is destroyed during testing; (b) the results need to be corrected for strain-rate dependence; (c) the modulus, E, can only be evaluated from the slope of the stress-strain curve which requires measurements at several increasing levels of the strain; and (d) the value of $E$ is difficult to determine from the slope of the stress-strain curve when the slope is rapidly changing as is the case for most tissues.

Because of the limitations of the constant rate-of-strain tests, a variety of new approaches have been developed to attempt to characterize the mechanical properties of tissues and implants. These include incremental stress-strain testing, magnetic resonance elastography, ocular response analysis, optical coherence tomography, ultrasound elastography, and surface wave optical coherence elastography. Below we compare these different approaches.

\subsection{Incremental Stress-Strain Testing}

This test is similar to constant rate of strain testing except a strain interval is applied to the sample in steps; between steps, the sample is allowed to relax until it reaches its final dimensions [28]. The value of this method is that it gives an elastic modulus from the equilibrium stress-strain curve that is a material property (not strain-rate dependent). But the test also requires destruction of the tissue and measurements at increasing strain values. Relaxation of the material to equilibrium at each step may require up to 24 hours and is a time consuming process.

\subsection{Magnetic Resonance Elastography (MRE)}

Low et al. [29] review the use of magnetic resonance elastography to calculate values of tissue modulus. In this method, mechanical excitation is produced by pneumatic, electromechanical, or piezoelectric stimulators positioned next to the body. The tissue is loaded by one of these means and then the MRI signal is collected. The phase shift in the MRI signal is used to calculate a value of the modulus; however, the workers assume that Poisson's ratio is 0.5 and that the tissue density is $1.0 \mathrm{~g} / \mathrm{cc}$. These assumptions create calculation errors since Poisson's ratio has been shown to vary from 0.5 for tissues $[19,28]$. The value of this technique is that it can be used noninvasively in real time; however, use of this technique requires correction for Poisson's ratio and strain-rate effects. 


\subsection{Ocular Response Analyzer (ORA)}

The ocular response analyzer is a clinical device that uses a high speed air puff to deform the cornea. Changes in shape of the anterior surface are tracked using an infrared beam reflected from the surface of the cornea and aligned with the geometry of a detector [30]. Using this device, corneal deformation is tracked after the air puff is applied to the corneal surface. Differences in the pressures between the inward and outward flattening of the cornea are reported as the corneal hysteresis. Changes in the corneal hysteresis are correlated with disease states anecdotally. The ability to make non-invasive measurements using this device is a positive attribute of this method. However, the inability to relate the results to standard mechanical testing parameters limits the utility of this device.

\subsection{Optical Coherence Elastography (OCE)}

Kennedy et al. [31, 32] have reviewed the use of optical coherence elastography for the analysis of tissue mechanical properties. This technique uses coherent infrared light that is reflected off the tissue surface and compares the reflected light to the non-reflected light to create an image. The shear displacement of the surface is determined after the tissue undergoes a small displacement. This technique is non-invasive and can be used to evaluate tissue in situ. However, the values of moduli obtained from the models used appear lower than those calculated from destructive testing [28, 31, 32], suggesting that the strains introduced are not large enough to deform the structural components of the tissue. In addition, this technique can only be applied to surface tissues.

\subsection{Ultrasound Elastography (UE)}

Drakonaki et al. [33] point out that ultrasound elastography is referred to by a number of terms including strain elastography, compression elastography, sonoelastography, and real-time elastography. Using these techniques a low frequency compression is applied to the tissue, frequently via a hand held transducer. The applied compression induces a shear strain and the modulus is estimated from the change in the echo before and after the force is applied. ZaleskaDorobisz et al. [34] review the use of ultrasound to calculate modulus values of tissues for different clinical applications. This technique assumes that the tissue is a linearly elastic solid that has a Poisson's ratio of 0.5 and does not measure the modulus directly.

\subsection{Surface Wave and Optical Coherence Tomography (OCT)}

Li et al. [35] report creation of a surface wave in the cornea and evaluation of the mechanical properties using surface wave velocity measurements. They use pulsed laser excitation to create a surface wave and estimate the modulus from an equation that relates the surface wave velocity to the modulus. Song et al. [36] use ultrasound to create a shear wave and used OCE to measure the properties of tissue. The above studies assume a value for Poisson's ratio and a density to calculate the mechanical properties.

Shah et al. [37] use vibrational analysis in concert with OCT to measure the natural frequency of decellularized dermis and silicone rubber. They apply an acoustic vibration to the samples under 
tension and show that the natural frequency squared obtained from the change in frequency of the reflected light from the sample was directly related to the tensile modulus obtained in an incremental stress-strain experiment. Moduli from vibrational analysis compare very well to moduli obtained from incremental stress-strain curves. Their method does not rely on the assumption of a value of Poisson's ratio.

Non-destructive and non-invasive characterization of tissues and implants has been an important goal for researchers for decades. Unfortunately, the use of ultrasound and elastography provide only estimates of the exact values of mechanical parameters such as the modulus. OCE has been recently applied to studying tissue properties in health and disease; however, the values reported for tissue moduli are in the $\mathrm{kPa}$ range $[31,32]$ as opposed to the MPa range that is expected for biological polymers [28, 37-47].

The purpose of this paper is to present measurements of the elastic modulus of tissues in vivo along with measurements made on synthetic polymeric materials in vitro using vibrational OCT. Using this approach a spectrum of resonant frequencies and moduli are obtained by analyzing the frequencies at which the maximum displacements are observed for both tissues and synthetic polymers.

\section{Materials and Methods}

\subsection{Materials}

Tests were conducted on 15 volunteers with ages ranging from 24 to 70 years old after informed consent was obtained. All studies were conducted at $75{ }^{\circ} \mathrm{F}$ and $40 \%$ to $50 \%$ relative humidity. Tissues studied in vivo include bone, cartilage, fat, fibrotic tissue, ligaments, meniscus, nerve, skin, ocular tissues, tendon and vascular tissues as described in Table 1. All reported resonant frequencies and moduli are averages from at least 5 different individuals or specimen samples and each measurement on a specific sample was repeated three times.

Polymeric materials including silicone rubber, Viton rubber, butyl rubber, and acrylonitrile butadiene styrene (ABS) were used in this study. Silicone rubber sheets were produced by mixing polydimethylsiloxane base material with curing agent and allowing the mixture to harden in a 100 $\mathrm{mm}$ diameter petri dish. Polydimethylsiloxane (PDMS, silicone) samples were fabricated from a 2part kit (Corning Sylgard-184) by mixing silicone base and curing agent in a ratio of 10:1. Butyl rubber sheet was obtained from Discount Rubber Direct and an ABS coupling was obtained from World Class Plastics. Viton rubber gaskets were obtained from Blaylock Gasket. Measurements made on synthetic polymers were obtained from tensile stress-strain experiments and VOCT analyses in vitro. 
Table 1 Resonance Frequency and modulus values for human tissues are shown determined from vibrational optical coherence tomography. Note the values of the resonant frequency were determined as the frequency at which the maximum displacement of the tissue occurs. The modulus was calculated from equation (1a) based on stress-strain testing and vibrational measurements.

\begin{tabular}{|c|c|c|}
\hline Tissue & Resonant Frequency $(\mathrm{Hz})\{\mathrm{SD}\}$ & Modulus E (MPa) \{SD\} \\
\hline \multicolumn{3}{|l|}{ Bone } \\
\hline Lamellar Bone & $990\{10.0\}$ & $173\{20.0\}$ \\
\hline Subchondral Bone & $586\{26.07\}$ & $67.81\{11.11\}$ \\
\hline Ear and Lower Nasal cartilage & $290\{14.14\}$ & $16.2\{1.74\}$ \\
\hline Upper Nasal Cartilage & $380\{14.14\}$ & $30.4\{5.89\}$ \\
\hline Fat, Epidermal Cells & $40-70\{12.90\}$ & $1.110\{0.25\}$ \\
\hline Dermal Fibrotic Tissue & $210\{10.0\}$ & $10.84\{2.48\}$ \\
\hline \multicolumn{3}{|l|}{ Ligament } \\
\hline Anterior Cruciate Ligament (ACL) & $525\{7.07\}$ & $53.9\{2.25\}$ \\
\hline Meniscus & $430\{14.14\}$ & $31.4\{3.37\}$ \\
\hline \multicolumn{3}{|l|}{ Muscles } \\
\hline Bicep Muscle & $378\{16.02\}$ & $29.6\{2.62\}$ \\
\hline Quadriceps Muscle & $365\{21.21\}$ & $20.5\{2.32\}$ \\
\hline \multicolumn{3}{|l|}{ Nerve } \\
\hline Radial, Medial Nerve & $266\{11.54\}$ & $15.86\{2.24\}$ \\
\hline Normal Skin & $110\{7.38\}$ & $2.15\{0.29\}$ \\
\hline \multicolumn{3}{|l|}{ Ocular } \\
\hline Cornea, Sclera & $140\{14.14\}$ & $2.4\{0.14\}$ \\
\hline \multicolumn{3}{|l|}{ Tendon } \\
\hline Achilles Tendon & $440\{10.0\}$ & $34.0\{5.98\}$ \\
\hline Flexor Digitorum Profundus Tendon & $370\{14.14\}$ & $22.7\{9.42\}$ \\
\hline Patellar Tendon & $430\{5.77\}$ & $33.8\{4.62\}$ \\
\hline \multicolumn{3}{|l|}{ Vascular } \\
\hline Carotid Artery & $136\{11.54\}$ & $4.64\{0.98\}$ \\
\hline Radial Artery & $155\{11.98\}$ & $3.66\{0.65\}$ \\
\hline Vein & $165\{7.07\}$ & $4.84\{0.025\}$ \\
\hline
\end{tabular}

\subsection{Vibrational Optical Coherence Tomography OCT and Vibrational Analysis in Vivo}

VOCT is a non-invasive and non-destructive method that uses infrared light and audible sound to create a displacement of skin as described in detail previously [37-47]. The displacement of the skin causes vibrations in subcutaneous tissues that are reflected back to the skin. The result is a spectrum of resonant frequencies that are recoded by measuring the displacement of the skin as a function of frequency. The measured resonant frequencies are converted into modulus values using calibration equations (equations 1a-3) developed based on in vitro uniaxial mechanical tensile testing and VOCT measurements [37-47]. 
Experimentally, the VOCT hand piece uses infrared light to image the specimen as described previously [37-47]. The infrared beam is focused on a fixed position on the area of skin above the tissue to be studied. A frequency generating app is downloaded onto the i5 processor within the OCT device. This app is capable of driving the speaker between 30 and 20,000 Hz [37-47]. The speaker is placed near but not touching areas of the skin or polymer sample. During in vivo measurements, no sensation of the light or sound is felt impinging on the skin.

An OCT scanning image of the skin is obtained to insure that the epidermis and dermis are normal [37-47]. The spectral-domain optical coherence tomography (SD-OCT) system uses a fibercoupled superluminescent diode light source with an $810 \mathrm{~nm}$ center wavelength and $100 \mathrm{~nm}$ bandwidth (full-width at half maximum) [37-47]. Although the infrared light only penetrates about 0.5 to $1 \mathrm{~mm}$ into the skin, the audible sound will penetrate about $8.0 \mathrm{~cm}$ through the subcutaneous tissues. Subcutaneous tissues are identified from images obtained using ultrasound imaging at a frequency of $7.5 \mathrm{MHz}$. For measurement of the resonant frequency of a material the OCT system is operated in the B mode with the beam focused on one point with an area of about $14 \mu \mathrm{m}$ as previously indicated [37-44].

The resonant frequency of each sample is determined by measuring the displacement of skin resulting from sinusoidal driving frequencies ranging from $30 \mathrm{~Hz}$ to $20,000 \mathrm{~Hz}$, in steps of $10 \mathrm{~Hz}$ for tissues and rubber samples. Steps of $200 \mathrm{~Hz}$ were used for samples of ABS. The peak frequency (the resonant frequency), $f_{n}$, is defined as the frequency at which the displacement is maximized as discussed previously [37-47]. The moduli of skin and other tissues listed in Table 1 are calculated from measurements of $f_{n}$ and using equation (1a) for tissues. These measurements were made in vivo after the tissues were identified using high frequency ultrasound. The moduli of slicone, Viton and butyl rubber were calculated from equation (2) and ABS from equation (3) where $\mathrm{E}$ is in $\mathrm{Pa}$ (Pascals) and $\mathrm{d}$, the thickness, is in meters. These measurments were made in vitro using results of stress-strain and VOCT analyses.

The material's modulus $(E)$ determined from these vibrational measurements is related to the resonant frequency $f_{n}$ through equation (1) where $m, L$, and $A$, are the mass, length, and crosssectional area of the sample, respectively as discussed previously [37-44].

$$
E=m\left(2 \pi f_{n}\right)^{2}\left(\frac{L}{A}\right)
$$

Moduli determined using equation (1) were plotted against the corresponding moduli obtained from the tangent to the tensile stress-strain curves for all materials tested as previously discussed [37-44] and were used to develop equation (1a) for tissues and equations (2) and (3) for rubbers and $A B S$, respectively. The differences in equations (1a)-(3) arise due to the fact that the ratio of mass divided by the cross sectional area varies for different materials. In other words the differences in equations (1a), (2) and (3) arise due to differences in material density. Since most soft tisues have a density very close to 1.0 equation $1(\mathrm{a})$ is valid for the majority of tissues found in the body.

$$
\begin{array}{ll}
\text { Tissues } & E * d=0.0651 *\left(f n^{2}\right)+233.16 \\
\text { Rubbers } & E * d=0.3106 *\left(f n^{2}\right)-1145.1 \\
\text { ABS } & E * d=5.904 *\left(f n^{2}\right)-4 *\left(10^{7}\right)
\end{array}
$$

The skin thickness is determined from the OCT image and the polymer thickness is measured using a calipers. The thickness is used for determination of the modulus in equations (1-3) The weighted displacement versus frequency curve becomes a mechanical and vibrational spectrum 
generated by the components of the tissues or polymeric samples that are vibrated [37-47]. Weighted displacement is normalized by dividing by the displacement of the speaker in the absence of the sample to correct for the displacement of the speaker as a function of frequency.

\section{Results}

Using VOCT it is possible to measure the resonant frequency and calculate the modulus for natural and synthetic polymers using non-invasive and non-destructive methods. While tissues are composed of a complex mixture of cells, macromolecules and supramolecular organizations of collagen fibrils and fibers, it is possible to identify individual components based on the measured values of resonant frequency and modulus. Figure 1 shows a plot of weighted displacement versus frequency for skin from the top of the hand and skin from the wrist over the radial artery. Both plots show that there are peaks at different frequencies. Skin from the top of the hand shows a peak at about $120 \mathrm{~Hz}$ versus peaks of $70 \mathrm{~Hz}, 100 \mathrm{~Hz}$ and $150 \mathrm{~Hz}$ from skin over the radial artery The moduli associated with the latter peaks are $1.0 \mathrm{MPa}(70 \mathrm{~Hz}), 2.0 \mathrm{MPa}(100 \mathrm{~Hz})$ and about 3.66 period MPa $(150 \mathrm{~Hz})$. The mean resonant frequencies measured and moduli calculated from equations (1-3) are listed in Table 1 . The tissues cited were identified by ultrasound imaging in vivo and the resonant frequencies were measured using VOCT in vivo. The values for the polymers listed in Table 1 were determined in vitro from stress-strain testing and VOCT measurements.

Figure 2 shows plots of weighted displacement versus frequency for patellar and Achilles tendons. While patellar tendon shows peaks at 100,300, 380 and $440 \mathrm{~Hz}$, Achilles tendon has a single major peak at $440 \mathrm{~Hz}$. The modulus associated with the peak at $440 \mathrm{HZ}$ is $34 \mathrm{MPa}$. The additional peaks in the plot for patellar tendon are due to other subcutaneous tissue components that are identified from Table 1 and ultrasound imaging after testing a spectrum of other tissues. The peaks at 300 and $380 \mathrm{~Hz}$ appear to be due to cartilage and muscle, respectively, that are in close proximity to the patellar tendon; however, the prominent peak $(440 \mathrm{~Hz})$ is from patellar tendon.

Figure 3 shows a plot of weighted displacement versus frequency for a new Viton rubber gasket and one for a gasket (old gasket) that has been in service in a transmission for 100,000 miles. Note the broadness of the peak for both gaskets and the reduction in the resonant frequency after fatigue cycling of the material. The resonant frequency and modulus of the new gasket are $180 \mathrm{~Hz}$ and $11.45 \mathrm{MPa}$, respectively and that after use are reduced to $140 \mathrm{~Hz}$ and $5.99 \mathrm{MPa}$. After exposure to high temperatures and pressures the modulus is significantly reduced and the peak is shifted to the left. In addition, the right hand portion of the peak is lost whereas the left hand portion appears to remain intact. This suggests that the molecular weight of the constituent crosslinked polymer chains appears to be reduced during cycling of the material at high temperatures. The weighted displacement versus frequency profile appears to reflect the molecular weight distribution of the constituent polymer chains in the crosslinked sample. 

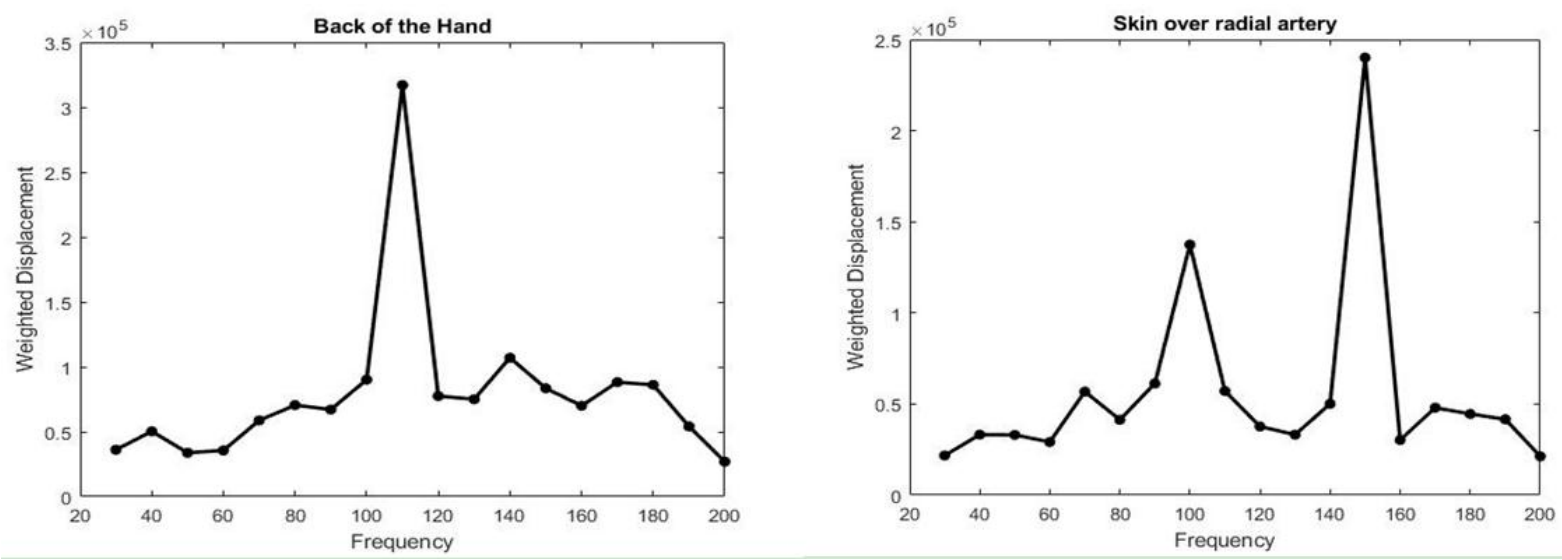

Figure 1 Weighted displacement versus frequency for human skin from the top of the hand (left) and skin over the radial artery from the wrist (right) determined using VOCT. Note the skin from the hand has a single resonant frequency of about $100 \mathrm{~Hz}$ representing the collagen in the papillary dermis. The resonant frequency is defined as the frequency at which the maximum displacement is observed. Skin over the wrist has resonant frequencies of 70,100 and $150 \mathrm{~Hz}$ reflecting the cells in the epidermis $(70 \mathrm{~Hz})$, collagen in the papillary dermis $(100 \mathrm{~Hz})$ and the extracellular matrix found in the arterial wall $(150 \mathrm{~Hz})$.
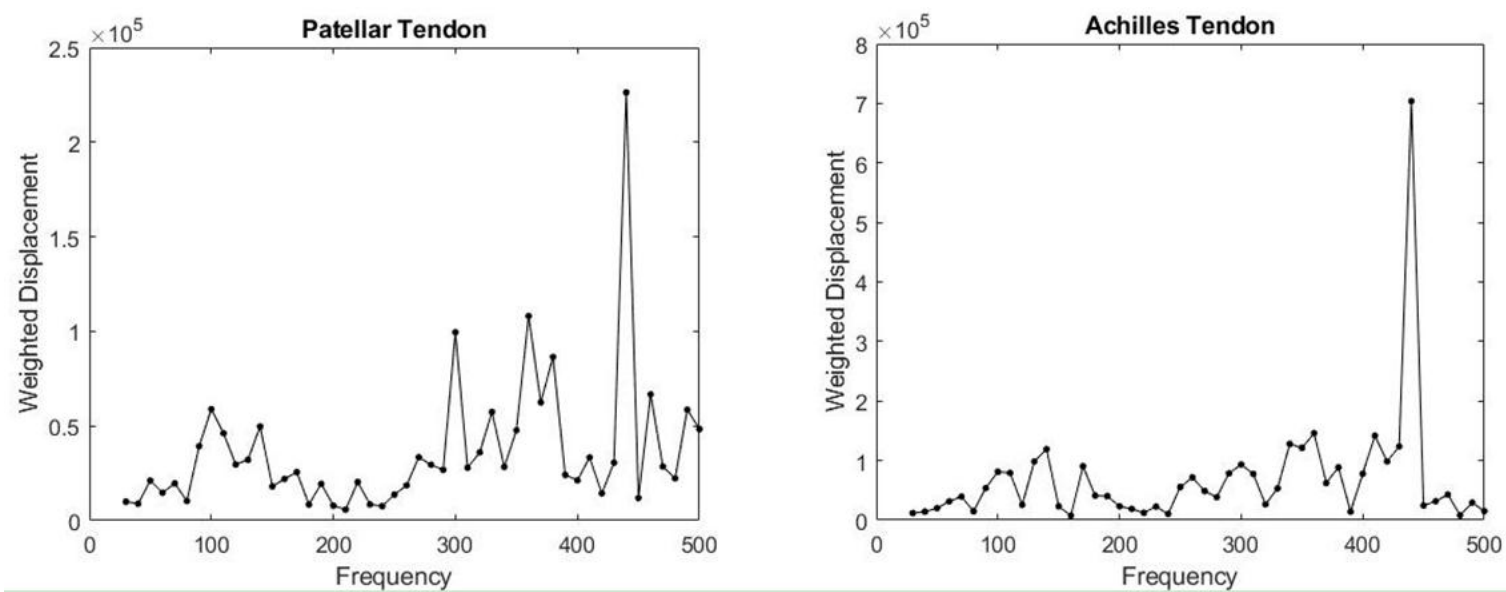

Figure 2 Weighted displacement versus frequency for human patellar tendon (left) and Achilles tendon (right) determined using VOCT and high frequency ultrasound imaging. Note the patellar tendon has major peaks at 100,300, 380 and $440 \mathrm{~Hz}$ while the Achilles tendon has a single major peak at a frequency of about $440 \mathrm{~Hz}$ representing aligned collagen fibers. The resonant frequency is defined as the frequency at which the maximum displacement is observed. The peaks at 300 and $380 \mathrm{~Hz}$ seen in patellar tendon represent the surrounding cartilage and muscle near the patellar tendon based on the data reported in Table 1 and ultrasound images. 

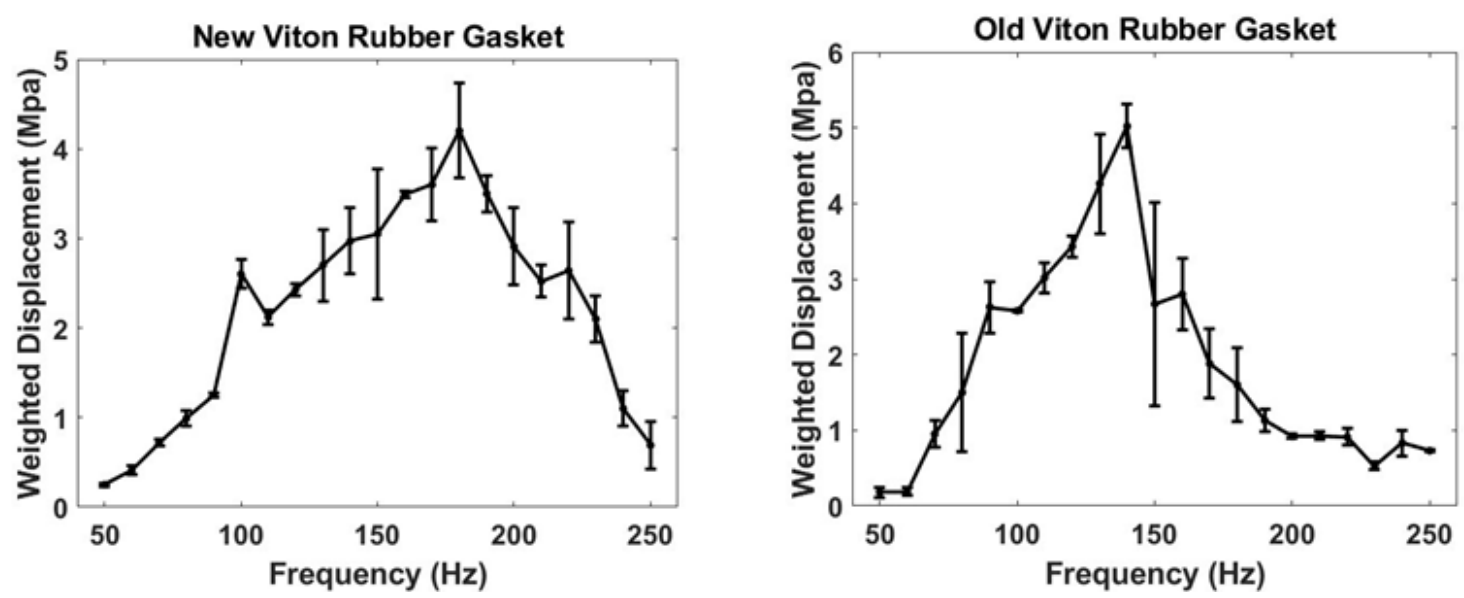

Figure 3 Weighted displacement versus frequency determined using VOCT for a Viton rubber gasket before (new-left) and after (old-right) use in an automotive transmission. The resonant frequency is defined as the frequency at which the maximum displacement is observed. Note the resonant frequency of the old gasket is lower than that of the new gasket and the curve also shifts to lower frequencies. These data suggest that the resonant frequency curve may reflect decreases in the distribution of chain lengths that occur with aging and fatigue of the gasket.

Table 1 gives a summary of the resonant frequency and modulus data for the tissues studied including fat, epidermal cells, skin, scar, tendon, ligament, blood vessels, muscle, cartilage, meniscus, bone and nerve. The resonant frequencies vary from about $40 \mathrm{~Hz}$ (fat) to about $990 \mathrm{~Hz}$ (lamellar bone) and the moduli vary from about 1 to $173 \mathrm{MPa}$.

Table 2 shows a summary of the resonant frequency and modulus data for silicone, butyl rubber, new and old Viton rubber gaskets and $A B S$ determined from stress-strain measurements and VOCT. The resonant frequency and moduli vary from 120 to $2800 \mathrm{~Hz}$ and 2 to $2120 \mathrm{MPa}$, respectively.

Table 2 Resonant frequency and modulus measurements of synthetic polymers determined from vibrational optical coherence tomography. The resonant frequency is defined as the frequency at which the displacement is maximized. The moduli were calculated using equation (2) for rubbery materials and equation ( 3 ) for ABS based on both stress-strain testing and VOCT measurements.

\begin{tabular}{|l|l|l|}
\hline Sample & Resonant Frequency (Hz) \{SD $\}$ & Modulus E (MPa) \{SD\} \\
\hline ABS Plastic & $2800\{10.0\}$ & $2120\{0.02\}$ \\
\hline Silicone Rubber & $80\{10.0\}$ & $1.68\{0.23\}$ \\
\hline New Viton Gasket & $180\{5.0\}$ & $11.45\{0.64\}$ \\
\hline Old Viton Gasket & $140\{5.0\}$ & $5.99\{0.43\}$ \\
\hline
\end{tabular}

Figure 4 is a mechanical and resonant frequency spectrum for both tissues and synthetic polymers reported in this study. Note the resonant frequencies and moduli are a characteristic fingerprint of each tissue and polymeric material. Tissues can be characterized for their cellular and macromolecular content based on their individual profiles. 


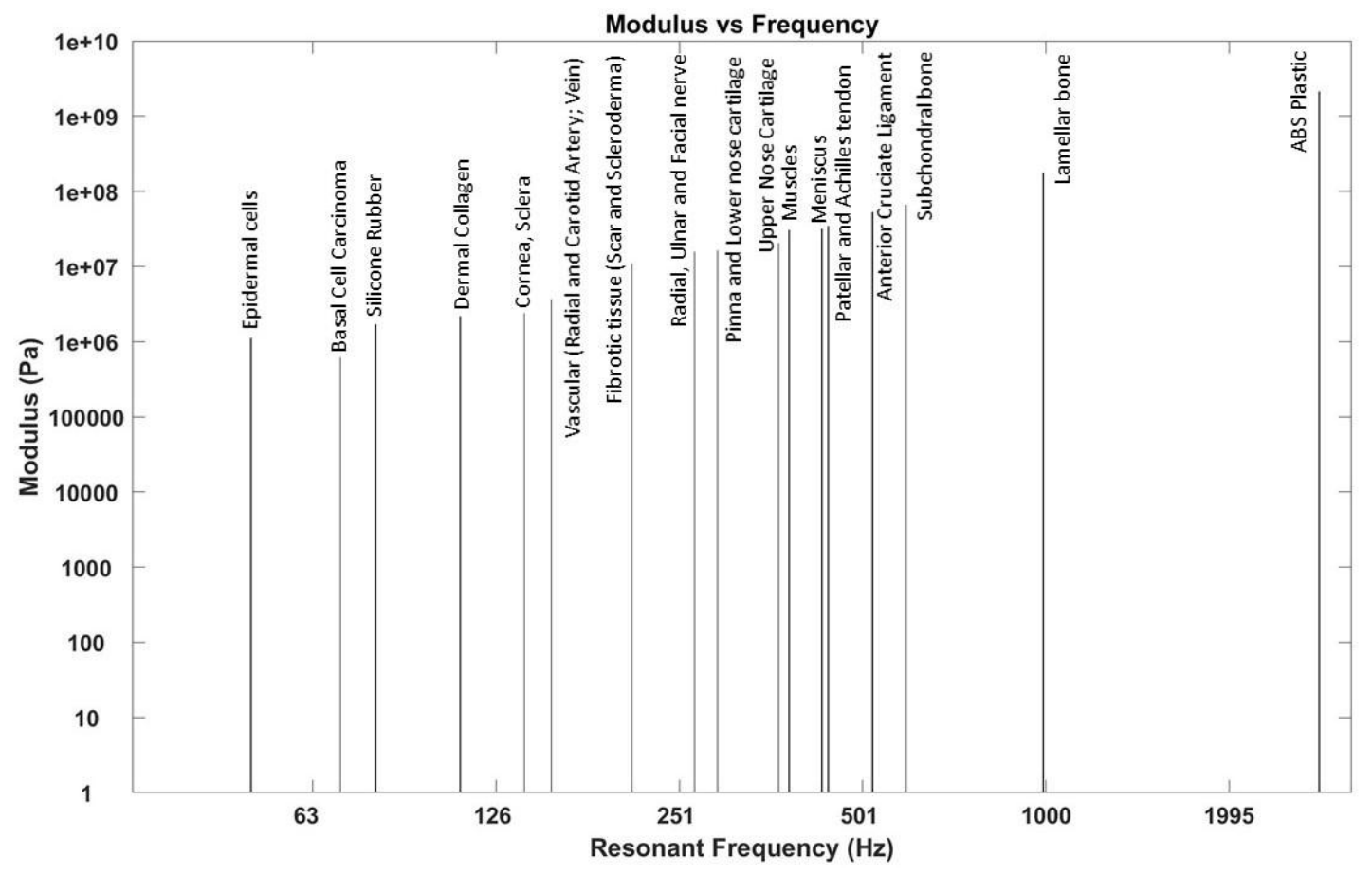

Figure 4 Elastic modulus versus resonant frequency of different tissues and polymers studied with VOCT. The resonant frequency of a variety of tissues was measured using VOCT and the moduli were calculated using equations (1a-3). The resonant frequency spectrum of each tissue or polymeric material is a characteristic of the macromolecules that make up the tissue, their concentrations, fiber diameters and the fiber orientation. The result of this is that collagen in the dermis has different properties from collagen in scar tissue and tendon due to the differences in organization and concentration.

\section{Discussion}

The ability to non-invasively and non-destructively characterize tissues and implants has been a goal of biomaterials workers for decades. The need to be able to (1) provide quality control testing on individual implants; (2) evaluate biocompatibility in cell and animal testing without destroying cell cultures or sacrificing animals prematurely; and (3) follow implant functioning in humans, has underscored the need for better tools for predicting success of materials used in implants. Factors that lead to implant failure such as vasodilation and bleeding surrounding the implant, modulus mismatches, and biodegradation can all be evaluated non-invasively using VOCT in vivo.

VOCT offers distinct advantages over many of the current methods used to characterize tissues and implants. The ability to make non-invasive and non-destructive measurements both in vitro and in vivo gives biomaterials workers additional tools to evaluate the effects of changing process parameters, material changes that occur during sterilization procedures, and time dependent outcome data in animals and humans. In addition, use of VOCT would decrease the number of animals used in biocompatibility studies since a single animal could be studied at multiple time points.

Our results suggest that VOCT studies on synthetic polymers are easier to interpret than measurements made on tissues. Synthetic polymers are characterized by a single broad peak 
(Figure 3). The broadness of the peak reflects the distribution of crosslinked polymer chain lengths that are reduced as mechanical fatigue occurs (Figure 3 new versus old Viton data). Measurement of these parameters could be made in vivo as an implant is biodegrading. This would assist with evaluating biodegradation rates of implants since the biodegradation rate of an implant in some cases may vary from person to person.

It is likely that the broadness of the peak seen in Figure 3, for Viton rubber, a synthetic polymer, reflects the molecular weight distribution. If this is the case then molecular weight distributions of uncrosslinked and crosslinked polymers could be determined rapidly with VOCT. The decreased peak width of the old Viton gasket compared to the new one indicates that higher molecular weight chains are broken down into lower molecular weight chains during fatigue. The lower molecular weight chains remain largely intact while the longer chains appear to be cleaved. Materials with moduli from $2 \mathrm{MPa}$ to over $2000 \mathrm{MPa}$ can be measured using VOCT (Table 2).

Biological materials are more complex than synthetic polymers since they are composed of mixtures of cells and materials with different molecular weights and physical properties. The interpretation of the weighted displacement versus frequency data is achieved by analyzing a variety of tissues containing different components. For cellular materials such as fat, there is one peak with a resonant frequency of about $40 \mathrm{~Hz}$ (see Table 1). Skin contains a cellular epidermal component and a collagenous dermis and is characterized by a cellular peak between 50 and $70 \mathrm{~Hz}$, and a dermal collagen peak between 100 and $120 \mathrm{~Hz}$ (Figure 1). Skin over the radial artery contains an additional peak at $150 \mathrm{~Hz}$ which is similar to that seen from other blood vessels. Other tissues such as ear, nose and joint cartilages have peaks at about $380 \mathrm{~Hz}$, muscle a peak at $350 \mathrm{~Hz}$, tendon and ligaments have peaks between 350 and $440 \mathrm{~Hz}$ and lamellar bone has a peak at $990 \mathrm{~Hz}$. By analyzing the peaks found in tissues with various combinations of components we are able to assign values of the resonant frequencies to the components of tissues as indicated in Figure 4. Figure 4 is a mechanical and frequency spectrum of the components found in tissues of the body. This figure can be used to evaluate tissue composition from a plot of weighted displacement versus frequency. It has been reported that the cellular and collagen resonant frequency peak widths are increased during skin wound healing [45-47] and in cancerous skin lesions [45-47] suggesting that the peak width may be an indicator of disease progression.

It has also been shown that the relative heights of the cellular and collagen peaks change in both benign and cancerous lesions [45-47] suggesting that cellular proliferation and the relative heights of the peaks give information on the composition of diseased tissue. In addition, the increase in the cellular stiffness seen in cancerous lesions [45-47] also gives information on cellcell interactions that may help to understand the pathophysiology of tissue changes that are associated with cancer. The ability to generate a mechanical and resonant frequency spectrum of a material is useful not only in identifying the components of normal tissue, but also is useful in analyzing the composition of tissue laid down in response to an implant or as a result of a disease such as cancer.

\section{Conclusions}

The characteristic spectrum of resonant frequencies and moduli of tissues and polymers used in implants can be measured using VOCT. The resonant frequencies of cells and tissues ranging from about $40 \mathrm{~Hz}$ to about $990 \mathrm{~Hz}$ can be measured using this technique. Increases in the resonant 
frequencies and moduli of tissues are reported to be associated with cancer and fibrosis of skin. For this reason VOCT is useful in analyzing changes in tissues that occur in disease and tissue surrounding implants. It is concluded that the width of the resonant frequency peak appears to be related to the molecular weight distribution and that VOCT may serve as a method to rapidly determine molecular weight distributions and fatigue in synthetic polymeric materials.

\section{Author Contributions}

Dr. Silver designed, interpreted the data and wrote the first draft of the paper. Dr. Horvath designed the ultrasound localization experiments used to identify the skin location of subcutaneous tissues. Nikita Kelkar and Tanmay Deshmukh collected the ultrasound and VOCT data and assisted in preparing the manuscript. Dr. Shah assisted in collecting and interpreting the VOCT data.

\section{Competing Interests}

The authors have declared that no competing interests exist.

\section{References}

1. Glasgold Al, Silver FH. Applications of biomaterials in facial plastic surgery. Boca Raton: CRC Press; 1991.

2. Silver FH, Christiansen DL. Biomaterials science and biocompatibility. N.Y.: Springer-Verlag; 1999.

3. Akbal C, Lee SD, Packer SC, Davis MM, Rin RC, Kaefer M. Bladder augmentation with acellular dermal biomatrix in a diseased animal model. J Urol. 2006; 176: 1706-1711.

4. Griffin JE, Johnson DL. Management of the maxillofacial burn patient: Current therapy. J Oral Maxillofac Surg. 2005; 63: 247-252.

5. Haddock N, Levine J. Breast reconstruction with implants, tissue expanders and alloderm: predicting volume and maximizing the skin envelope in skin sparing mastectomies. Breast J. 2009; 16: 14-19.

6. Gryskiewicz JM, Rohrich RJ, Reagan BJ. The use of alloderm for the correction of nasal contour deformaties. Plast Reconstr Surg. 2001; 107: 561-570.

7. Wax MK, Winslow CP, Andersen PE. Use of allogenic dermis for radial forearm free flap donor site coverage. J Otolaryngol. 2002; 31: 341-345.

8. Albo D, Awad SS, Berger DH, Bellows CF. Decelularized human cadveric dermis provides a safe alternative for primary inguinal hernia repair in contaminated surgical fields. Amer J Surg. 2006; 192: e12-e17.

9. Shorr N, Perry RD, Goldberg RA, Hoenig J, Shorr J. The safety and applications of acellular human dermal allograft in ophthalmic plastic and reconstructive surgery: A preliminary report. Ophthalm Plast Reconstr Surg 2000; 16: 223-230.

10. Harris RJ. Gingival augmentation with an acellular dermal matrix: Human evaluation of a caseplacement of the graft on periosteum. Int J Periodont Restorat Dent. 2004; 24: 378-385.

11. Lee JM, Boughner DR. Mechanical properties of human pericardium. Differences in viscoelastic response when compared with canine pericardium. Circul Res. 1985; 57: 475-481. 
12. McGuire DA, Hendricks SD. Allograft tissue in ACL reconstruction. Sports Med Arthr Rev. 2009; 17: 224-233.

13. Ward RM, Sung VW, Clemons JL, Myers DL. Paravaginal repair with an alloderm graft. Amer J Obstetr Gynecol. 2007; 197: c1-c5.

14. Altman GH, Diaz F, Jakuba C, Calabro T, Horan RL, Chen J, et al. Silk-based biomaterials. Biomaterials. 2003; 24: 401-416.

15. Bhomiratana S, Vunjak-Novakovic G. Concise review: Personalized human bone grafts for reconstructing head and face. Cells Transl Med. 2012; 1: 64-69.

16. Deekan CR, Lake SP. Mechanical properties of the abdominal wall and biomaterials utilized for hernia repair. J Mechan Behav Biomed Mater. 2017; 74: 411-417.

17. Silver FH. Biomaterials medical devices and tissue engineering, London, UK: Chapman Hall; 1994.

18. Silver FH. Mechanosensing and mechanochemical transduction in extracellular matrix. N.Y.: Springer; 2006.

19. Silver FH, Shah R. Measurement of mechanical properties of natural and engineered implants. Adv Tissue Eng Regen Med. 2016; 1: 1-9.

20. Samani A, Zubovits J, Plewes D. Elastic moduli of normal and pathological humanbreast tissues: An inversion-technique-based investigation of 169 samples. Phys Med Biol. 2007; 52: 1565-1576.

21. Acerbi I, Cassereau L, Dean I, Shi Q, Au A, Park C, et al. Human breast cancer invasion and aggression correlates with ECM stiffening and immune cell infiltration. Integr Biol. 2015; 7: 1120-1134.

22. Kalath S, Tsipouras $P$, Silver FH. Non-invasive assessment of aortic mechanical properties. Ann Biomed Eng. 1986; 14: 513-524.

23. Kalath S, Tsipouras $\mathrm{P}$, Silver FH. Increased aortic root stiffness associated with osteogenesis imperfecta. Ann Biomed Eng. 1987; 15: 91-99.

24. Lin HH, Lin HK, Lin IH, Chiou YW, Chen HW, Liu CY, et al. Mechanical phenotype of cancer cells: Cell softening and loss of stiffness sensing. Oncotarget. 2015; 6: 20946-20958.

25. Lekka M. Discrimination between normal and cancerous cells using AFM. Bionanoscience. 2016; 6: 65-80.

26. Fung YC. Biomechanics: Mechanical properties of living tissue. $1^{\text {st }}$ edn. New York: SpringerVerlag; 1973. pp.568.

27. Yamada H. Strength of biological materials. Baltimore: Williams and Wilkins; 1970. pp.297.

28. Dunn MG, Silver FH. Viscoelastic behavior of human connective tissues: Relative contribution of viscous and elastic components. Conn Tis Res. 1983; 12: 59-70.

29. Low G, Kruse SA, Lomas DJ. General review of magnetic resonance elastography. World J Radiol. 2016; 8: 59-72.

30. Ruberti JW, Roy AS, Roberts CJ. Corneal biomechanics and biomaterials. Annu Rev Biomed Eng. 2014; 13: 269-295.

31. Kennedy BF, Kennedy KM, Sampson DD. A review of optical coherence elastography: Fundamentals, techniques and prospects. IEEE J Sel Top Quant Electr. 2014; 20: 272-288.

32. Kennedy BF, McLaughlin RA, Kennedy KM, Chin L, Curatolo A, Tien A, et al. Optical coherence micro-elastography: Mechanical-contrast imaging of tissue microstructure. Biomed Opt Express. 2014; 5: 2113-2124. 
33. Drakonaki EE, Allen GM, Wilson DJ. Ultrasound elastography for musculoskeletal applications. Br J Radiol. 2012; 85: 1435-1445.

34. Zaleska-Dorobisz U, Kaczorowski K, Pawlus A, Puchalskoc A, Inglot M. Ultrasound elastographyreview of techniques and its clinical application. Adv Clin Exp Med. 2014; 23: 645-655.

35. Li C, Guan G, Huang Z, Johnstone M, Wang RK. Noncontact all-optical measurement of corneal elasticity. Opt Lett. 2012; 37: 1625- 1627.

36. Song S, Le NM, Huang Z, Shen T, Wang RK. Quantitative shear-wave optical coherence elastography with a programmable phased array ultrasound as the wave source. Opt Lett. 2015; 40: 5007-5010.

37. Shah R, Pierce MC, Silver FH. A method for non-destructive mechanical testing of tissues and implants. J Biomed Mater Res A. 2016; 105: 15-22.

38. Silver FH, Shah R. "Virtual biopsies" of normal skin and thermal and chemical burn wounds. Adv Skin Wound Care. 2020; 33: 1-6.

39. Silver FH, Silver LL. Gravity. Mechanotransduction and healing: How mechanical forces promote tissue repair. SM J Biomed Eng. 2017; 3: 1023.

40. Silver FH. A matter of gravity-mechanotransduction: How mechanical forces influence biological materials. Mater Sci Eng Int J. 2017; 1: 00012.

41. Silver FH, Silver LL. Non-invasive viscoelastic behavior of human skin and decellularized dermis using vibrational OCT. Derm Clin Res. 2017; 3: 174-179.

42. Silver FH, DeVore D, Shah R. Biochemical, biophysical and mechanical characterization of decellularized dermal implants. Mater Sci Appl. 2017; 8: 873-888.

43. Silver FH, Silver LL. Use of vibrational optical coherence tomography in dermatology. Arch Dermatol Skin Care. 2018; 1: 03-08.

44. Silver FH, Shah RG, Benedetto D, Dulur A, Kirn T. Virtual biopsy and physical characterization of tissues, biofilms, implants and viscoelastic liquids using vibrational optical coherence tomography. World J Mech. 2019; 9: 90332-90348.

45. Silver FH, Shah RG, Richard M, Benedetto D. Comparative "virtual biopsies" of normal skin and skin lesions using vibrational optical coherence tomography. Skin Res Tech. 2019; 25: 743-749.

46. Silver FH, Shah RG, Richard M, Benedetto D. Comparison of the virtual biopsies of a nodular basal cell carcinoma and an actinic keratosis: Morphological, cellular and collagen analyses. Adv Tissue Eng and Regen Med. 2019; 5: 61-66.

47. Silver FH, Shah RG, Richard M, Benedetto D. Use of vibrational optical coherence tomography to image and characterize a squamous cell carcinoma. J Derm Res Ther. 2019; 5: 067. 


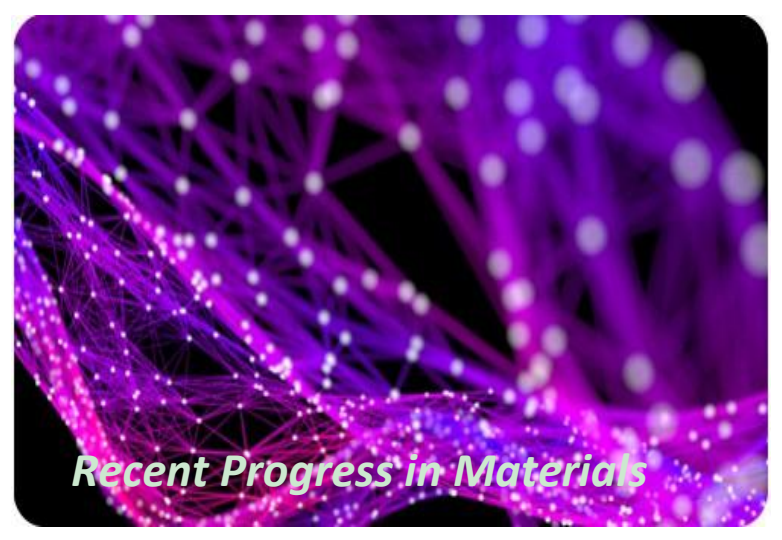

Enjoy Recent Progress in Materials by:

1. Submitting a manuscript

2. Joining in volunteer reviewer bank

3. Joining Editorial Board

4. Guest editing a special issue

For more details, please visit:

http://www.lidsen.com/journals/rpm 